\title{
KNOWLEDGE TO MANAGE THE KNOWLEDGE SOCIETY: COMPLEXITY AND THE SYSTEMIC CONCEPT OF CRISIS
}

\author{
Author(s) / Auteur(s) : \\ Gianfranco MINATI \\ Mathematician, Systems Scientist \\ President of the European Union for Systemics (UES) \\ President of the Italian Association for Systemic Research (AIRS) \\ gianfranco.minati@airs.it
}

\begin{abstract}
Résumé :
We briefly propose a possibly more precise systemic understanding of the process of crisis with the purpose of allowing suitable, appropriate modifying interventions. Examples and types of crises are introduced. At a suitable level of representation we consider crisis as a non-autonomous parasitic process of the hosting one(s); processes acquiring characteristics autonomous with respect to those of the hosting process(es); processes converging to degeneration and malfunctioning; which are emergent and given by coherent, subsequent and related new degenerative properties or loss of coherence among emergent processes of the hosting one(s). Possible symptoms for diagnostics and prediction of processes of crisis are outlined. Types of crisis are considered and some generic exemplifying types of actions on crises are proposed. The main purpose of this article is to show that different types of processes of crisis having different natures are possible and that suitable, appropriate approaches should be adopted.
\end{abstract}

\section{Keywords / Mots-clés :}

actions, coherence, complex, correlation, crisis, level

\section{INTRODUCTION}

This article intends to clarify theoretically the concept of crisis. Furthermore, possible types of crisis are introduced. A proper understanding of them may allow more effective and appropriate, possibly preventative or ongoing actions and approaches to deal with crises, whereas typical interventions are $a$ posteriori, oriented to recover and reduce the final negative or intermediate implications often through an assumption of linearity.

The more common understanding of crisis relates to situations where degeneration occurs possibly through sudden or successive negative events. The concept of crisis possesses an intrinsic negative generic meaning related to the fact that it usually applies to phenomena having social impact perhaps of an economic, educational, medical, military, philosophical, political, psychological, or sociological nature, rather than relating to the natural sciences such as physics where conceptually corresponding phenomena such as the occurrence of complex dynamics between classic and quantum regimes in phase transitions and symmetry breaking have been studied and exhaustively understood theoretically (Solé, 2011).

This article addresses a readership interested in the negative generic meaning used when dealing with the social cases mentioned above. The purpose is to ensure, through a more precise theoretical understanding of the concept of crisis, suitable interventions as presented here from a conceptual and methodological point of view postponing future possibly related quantitative models suitable for simulations. Some concepts relating to crises typical of the science of complexity should be transformed into culture, knowledge to manage the knowledge society (Kumar, 2004) which today still uses the knowledge of industrial society (Haunss, 2015). The knowledge of industrial society was suitable for dealing with pre-complexity problems. The knowledge society is characterised by complex problems requiring newly appropriated knowledge such as the ability to suitably deal with 
loss of coherence and consider the conceptual framework of theoretical incompleteness (Minati, 2016a).

It is not matter of divulging but, rather, of making such concepts usable, for instance, by educators, entrepreneurs, managers and politicians, by inserting or updating current language with new available meanings corresponding closely to their original scientific meanings.

Within a series of articles dedicated to knowledge for managing the knowledge society (Minati, 2012a; 2012b; 2015) this article attempts this transformation for the concept of crisis.

\section{CRISIS}

The term crisis has a wide variety of different possible disciplinary meanings.

A first possible understanding of the concept of crisis occurs when within an on-going process under study one observes the occurrence of events such as anomalies, discontinuities or degenerations. The process deviates from its original path and the sequence of deviations is understood as the process of crisis.

In this regard, within a process a crisis can be considered as a sequence(s) of changes, for example, in the values of a variable over time, considered to be negative as they involve, for instance, degeneration, instability or collapse, leading, for instance, to malfunctioning, loss of coherence(s), exhaustion, loss of sustainability, and loss of convergence.

In this case crisis may be intended as a kind of process parasitic of the original ones.

Conceptually it is possible to distinguish between the process of crisis and the hosting parasitized process when the crisis consists of deviations from and anomalies in the original one.

For instance, sequences of expenses which are subtracted from revenues and their sequences constitute the crisis, increasing debt and leading towards bankruptcy.

The variable, subject to such negative changes may, partially or completely, directly represent the process under study, such as increasing debt, or indirectly relate to the processes being, for instance, statistical or acquired (e.g., emergent) or mesoscopic (see the sub-section Levels of representation) when considering properties of clusters of interrelated variables, when relating, for example, to the health of a person through vital physiological indicators.

A second possible concept of crisis occurs when single critical events take place, having as a consequence the activation of a process of crisis: an earthquake has the consequences of subsequent damage. This process of crisis superimposes, perturbs, or even replaces the on-going processes.

When non-stochastic, such subsequent changes constituting a crisis may be sufficiently correlated, coherent, to establish a process, for example, external coherent perturbations such as the irregular process of the gradual disintegration of a mountain or the depletion of an aquifer.

Both in this case of sequences of aftermaths activated by critical events, and in the case of sequences of changes, deviations from the original path considered above, we consider henceforth crises as processes of crisis being aftermaths or changes almost correlated. As we shall see, more sophisticated correlations, such as non-linear ones, establish autonomous processes of crisis including emergent processes.

The processes of crisis are specific in their intrinsic non-sustainability (since they lead to degeneration or malfunctioning of the hosting process) and in their belonging to the domains of negative coherences which degenerate and disintegrate hosting processes.

However, this does not mean that a process of crisis might not lead to a novel situation understandable later as a radical development, as a game changer. 


\section{EXAMPLES AND TYPES OF CRISES}

We now introduce some specific examples for a more complete understanding of the concept of crisis considered above.

One possible elementary understanding of the concept of crisis is the notion of malfunctioning usually attributed to devices having occasional or degenerative problems in their operation, i.e., crises in their functioning converging to situations of out of order as the final effect.

We also mention the concept of crisis as an increasing loss of equilibrium; a temporary crisis expected to allow resumption of the original properties; repetitive crises; exhaustion leading to a recovery of original properties or through the use of antagonistic actions to recover the original configuration.

Examples of crisis, understood as processes to be recovered are available in economics, the first example being the tulip mania, in March 1637 (Dash, 2019), medicine (asthma attacks or cardiovascular crises; or a failed healing process), social relationships (marriage crises), or in cognitive science (due to stress, burnout).

In economics, Crisis Theory has been introduced (see, for instance, Shaikh, 2016; Walby, 2015).

Other different disciplinary understandings of the concept of crisis exist, for instance, in philosophy (Bunge, 2001; Franzini, 2015) when considering the process of the increasing inadequacy of a philosophical concept to deal with new cultural and social situations. New situations may derive from criticism or the appearance of new problems arising, for instance, from new technologies or scientific discoveries, e.g., the concepts of life, mind, and time-space. In political science (Paxton et al., 2015) one can consider, for instance, the crisis of the Roman Empire or of Communism, as well as the crisis in the classical concept of freedom which now has to take into account new possibilities of intervening in society today and in communications allowing increasing manipulation of information, leading even to requests for redefining the concept of democracy (Diamond and Plattner, 2015; Minati, 2006; 2016b).

At this point one has to consider the difference, if any, between the concept of crisis and that of catastrophe, often intended as a sudden and widespread disaster. A first difference is given by the fact that the concept of crisis has the nature of a process whereas the concepts of catastrophe and disaster have a prevalent nature of effects.

Catastrophe may be considered as one of the possible results of a process of crisis.

One example of a technical understanding of catastrophe is that of catastrophic learning in Neural Networks (Haykin, 2008; Pessa and Penna, 1995).

Catastrophic learning occurs when a neural network which has learned a training set of items, subsequently learns a new item where the result is usually a 'catastrophic forgetting' of the earlier items.

Catastrophe Theory was introduced by René Thom in the 1960s (Saunders, 2010).

In fact, small changes in the values of certain parameters of a non-linear system can cause equilibriums to appear or disappear along with their dynamics producing sudden, significant changes in the behaviour of the system as occurs in chaotic systems (Rasband, 2015). Catastrophe Theory studies the various ways in which system response can change at a bifurcation point (change of attractors) whereas chaos theory studies the sensitivity of a system to its initial conditions.

Catastrophe Theory allows such sudden changes intended as bifurcation points to be represented on well-defined geometrical structures (change of attractors).

In short, this theory considers a catastrophe as a critical degenerate (or irregular) point, stationary or singular, on a smooth, universally differentiable surface, where points are radical bifurcations in the behaviour of the system.

Catastrophe Theory, although suitable for modelling processes of morphogenesis, is finalised, whereas the concept of crisis specifies a generic kind of converging or non-converging process assumed to degenerate the initial one. We may say that morphogenesis is a particular type of crisis. 
Disaster relates to a judgement about the new resultant post-crisis or post-catastrophe configurations or effects.

Another example is given by the concept of crisis in physics, having a large number of well-studied related cases such as phase transitions, criticality, and meta-stability (Solé, 2011). We should also consider cracks within stressed materials reaching critical points of crisis leading to larger cracks or breakage (Yang, 2016).

\section{Non autonomous crises: sequences of linear or uncorrelated deviations}

We consider here the case where subsequent changes, deviations within current process(es), have the same correlation as the parasitized process(es). In this case deviations are linear. A particular case occurs when deviations are constant or have a regular rate, e.g., constant payment rate, or regularly increasing up to an end, e.g., pains during childbirth.

Another case occurs when subsequent changes, deviations within current process(es) constituting the crisis, are uncorrelated. In this case changes or deviations are uncorrelated events, i.e., they constitute only sequences and it is not possible to speak of processes.

Examples include sequences of robberies occurring in a town, degenerative events occurring within abandoned sites, percentages of energy spent in mechanical processes due to friction assumed to be irregularly degenerative and the stable, increasing or irregular sequence of percentage of money spent due to bureaucracy or gambling addiction.

\section{Crisis as an autonomous process: sequences of correlated changes}

We consider here the case where a crisis consists of subsequent correlated changes which establish a process corresponding non-linearly to the parasitized hosting one(s).

Here, deviations, or changes from the hosting dynamics acquire their own specific evolution, e.g., changes may increase in intensity, succeed one another with increasing speed, slow down or acquire non-linear sequences. However, the properties of such evolution need to be acquired case by case being context sensitive.

An interesting case occurs when the autonomous evolution of crisis processes which are parasitic of the current ones, as considered in the previous case, exhibits similar characteristics or properties. The crisis process can be considered autonomous from the hosting one(s) when it is possible to study its sequences independently, i.e., as a generic type of process to be investigated per se, without contextual references. This opens up the possibility of studying types of crisis in the same way as Systems Science studies system properties independently from the systems possessing or acquiring such properties (Minati and Pessa, 2006; 2018). In this view, the same type of crisis may apply to different processes and cases, but can be suitably treated with the same approaches.

We may consider types of crisis as being given by their characteristics, for instance, whether they are convergent in different ways e.g., linear, non-linear, or stochastically leading to a final, ideal or real, catastrophic event.

Further types of crisis may relate to the same possessed or acquired systemic properties, such as the reduction of openness or reduction of coherence for processes of emergence.

Considering various types of crisis also allows one to better recognise them.

\section{Crises as autonomous processes acquire their own identities and forms of independence from the original, hosting, parasitized one(s)}

Single or multiple crisis processes may occur, with or without correlations between them, both autonomous and non-autonomous, such as a respiratory crisis with changes in body temperature.

Autonomous crisis processes may combine or interfere, both in linear and non-linear ways, with the hosting process or even superimpose it until, possibly and at different levels, replacing it leading to degenerative or transformative transitions. 
Autonomous crisis processes may develop on their own, acquiring emergent properties.

There are also processes of diffusion of crises, when crisis processes may be contagious, and infect, through their malfunctioning, other interrelated processes. Conversely, this would allow one to talk of the localisation of crises.

The term systemic crisis refers to cascading effects due to interdependencies in a system, such as those of markets or finance, where the crisis or failure of single entities or clusters of entities can diffuse, one elementary example being positive feedback. The term systematic risk relates to 'simultaneous', unavoidable, recurrent impact upon components of a system, e.g., firms, due to the same perturbing effects, for example, earthquakes, terrorism or war.

\section{The case of coherence}

We consider here the particular case of crisis as an autonomous process where deviations, changes in hosting dynamics, acquire coherence as a particular case of those considered in the above sub-section Crisis as an autonomous process: sequences of correlated changes. An initial localised crisis regarding one aspect of the hosting process then leads to cascades of other aspects subsequently acquiring their own coherence, e.g., long-range correlation, falling within the basin of an attractor, or following power laws. Examples include the emerging coherence of organised crime or the increasing malfunctioning of a device.

Conversely, we may consider the case of a crisis possessing the same coherence of its hosting process, but leading in different ways to the loss of such coherence. Coherence is maintained but in such a decreasing way as to lead to its disintegration. One example is given by a swarm where inter-agent distances, for some reasons, increase in such a way as to lead to the disaggregation of the swarm. Analogous examples exist in economics.

\section{Changes as non-crises, non-processes}

We should consider when change can be suitably represented, considered as a process, as a case of a crisis process.

A generic understanding of the term process lies in its being a sequence of interrelated steps, procedural, with phases, such as development in biology. It is possible to speak of processes of adaptation, aging, assessment, learning, or recovery.

At this point we may ask whether any transformation, or transition, should be considered as a crisis process. Or conversely, which changes do not occur through crises. When is change not a process?

We can consider as emblematic of a non-process change, but rather as an instantaneous change, in physics the Curie point (Biswas et al. , 2014), the critical temperature at which certain materials lose their permanent magnetic properties, i.e., the intrinsic magnetic moments of the components of a material change their direction. More generally, when criticalities (situations at the boundary between ordered and disordered dynamical phases) or metastability (ability to maintain or switch between states due to small fluctuations), occur (Christense and Moloney, 2005).

It is matter of changes occurring as unique events, the change in its entirety rather than activating processes of subsequent changes converging to the overall change, such as a catastrophe. Such changes include:

- the case of so-called intrinsic or radical emergence (Licata, 2008; 2010; Licata and Minati, 2016). Examples include phase transitions (from water to ice, from paramagnetic to magnetic phases);

- spontaneous symmetry breaking considered in Quantum Field Theory (acquisition of superconductivity, superfluidity and protein-folding);

- the constitution of morphological catastrophic patterns (due, for instance, to volcanic eruptions and earthquakes); 
- the formation of temporary dissipative structures, dynamically stable far from equilibrium, due to the dissipation of matter and energy, such as whirlpools and hurricanes.

We can also consider chaotic systems such as the climate, very dependent upon initial conditions and rapidly changing attractors.

When change is recognised as being suitably represented as a crisis process then one needs to consider types of crises allowing for actions such as acceleration, avoidance, combination, enhancement, induction, modification, replication, deceleration, support, suspension, etc. of the crisis process.

In fact, the study of the crisis process is related to the theory of change (Pessa, 2008).

\section{Symptoms}

We consider here problems in the diagnostics and prediction of crisis processes, distinguishing between crisis processes involving single systems or collective interactive systems establishing communities.

In the case of single systems, one can consider the appearance of deviations, irregularities in current processes of the system under study. Such irregularities may concern linear processes, parametrical changes for non-linear processes, correlations among processes, and loss of coherence for emergent processes. Irregularities may vary, for instance, by type, by the way they recur, by their intensity, or by their correlation.

Correlated irregularities may finally converge to a resulting dominating general irregularity possibly having overall effects on the hosting process, such as a catastrophe.

Otherwise correlated irregularities may eventually acquire coherence and result in an emergent process of crisis. The autonomy of such emergent crisis processes however does not remove their fundamental parasitic nature of necessarily deriving from the hosting process(s).

In the second case, where collective interactive systems establish communities, their correlation properties provide a significant indicator.

It has been found, for instance, that before obvious symptoms of crisis appear within the community, correlations within that community increase and, at the same time, so does its variance (Gorbana, 2010). We recall that in statistics, variance is the expectation of the squared deviation of a random variable from its mean. It measures how far a set of random numbers is spread out from its mean.

Furthermore, once crisis processes reach their degeneration and converge towards their catastrophic end, a process may diverge into two different directions where either correlations and variance decrease, with concomitant recovery, or where correlations decrease but variance continues to increase, leading to a final catastrophic end (Gorbana, 2010).

\section{Levels of representation}

This sub-section concludes with a few comments regarding levels of representation. The temporal and spatial level should be selected by considering the appropriateness for the occurrence of significant processes of change and their concomitant results.

The level of representation relates to the adoption of micro-, macro- or mesoscopic levels (Buzsáki and Christen, 2016; Fannes et al., 1994; Liljenstrom and Svedin, 2005).

In short, at the microscopic level, constituent entities are considered as not being further reducible, such as individual vehicles in road traffic not being reducible to components such as wheels or engines. This level is of interest when considering, for instance, crises in traffic flow such as gridlocks.

At the macroscopic level, we consider the same, aggregated, microscopic entities and their properties, such as a gas consisting of microscopic entities (molecules) and macroscopic properties such as temperature, volume and pressure. At this level all microscopic information is lost. This level is of interest when considering, for instance, crisis of markets, e.g., recessions. 
At the mesoscopic level, we consider clusters of microscopic properties such as how many people within a building are using the stairs in any given instant regardless of whether they are ascending or descending or simply standing still on the stairs. At this level, not all microscopic information is lost, but rather clustered. This level is of interest when considering, for instance, crisis of niche markets, e.g., their dynamics of appearing and disappearing with effects on other markets.

A crisis may be invisible at one level of representation but visible at another.

As an example, consider the invisibility of a hypothetical flock established by artificial boid-like agents having very long spatial distances between them. Such a flock is not detectable from earth by the naked eye. Another hypothetical case occurs when the time interval among interacting agents, where one influences another's behaviour, for instance, through the exchange of information or energy, is very long when considered on the time scale of the observer as in geology.

Furthermore, processes of emergence are detectable by observers provided with a suitable cognitive system, considered in cognitive science (Friedenberg and Silverman, 2015; Nescolarde-Selva, 2014) as a system of interactions related to the affective and emotional sphere, attention, the inferential system, language, logical activity, memory, and perception. The experimental detection of phenomena of emergence, such as that of a flock, with its dynamical coherences, requires, for instance, suitable memory and inferential activity, in order to recognise a flock even when it is changing patterns, density, altitude, or direction. Although it may not be possible to cognitively, experimentally, detect a collective behaviour for scaling problems, it is possible to observe properties, such as mathematical ones, to be considered as characteristics, or symptoms, of collective behaviours such as the occurrence of attractors, bifurcations, chaos, coherence, network, power laws, scale invariance, and symmetry breaking properties.

Finally, we may ask whether the hosting process and the crisis process should necessarily have the same level of representation.

\section{ACTIONS ON CRISES}

After the considerations introduced above we can take into account types of intervention having different contextual suitability for the complexity level of the process of crisis under study. However, correspondences between types of crisis and types of action can only be indicative and not prescriptive or procedural since effectiveness depends mainly on context conditions and the modifying effects expected. The experimenter is required to contextualise depending on the nature of the process of crisis under study. As for complex problems, crisis processes (not always easily distinguishable from the hosting process) should be managed, i.e., channelled, hindered, and where possible induced, influenced, oriented, perturbed, decelerated or accelerated, or varied, rather than solved, i.e., converted, removed, stopped, or suspended.

\section{Actions on causes}

Causes are intended as being identified in constructivist ways, depending on the variables and models considered which are available to the observer. Constructivism, Cognitivism, and Cognitive Science (Friedenberg and Silverman, 2015), consider the observer as a generator of effective, i.e., with the power to act concretely, cognitive reality rather than of relativism. Overall, the cognitive strategy is to pass from trying to understand how something really is to finding out how it is more effective to think of it. For instance, a process may be considered as degenerative or transformational, an illness as biological or psychological, a system as complex or not, and a mathematical problem as being algebraic or geometrical. However, if on the one hand, the constructivist approach helps to conceive possible alternative options to understand cognitive reality, on the other, it also supports a multiple understanding by substituting the option or with and, even though to different contextualised degrees (see the case of the DYnamic uSAge of Models (DYSAM) in Minati and Pessa, 2006, pp. 64-75).

Causes are considered to originate crises and assumed to be identifiable. However, any internal fluctuations or external perturbations sufficiently intense and compatible with the on-going processes may give rise to a process of crisis. This can happen, for example, when fluctuations or external perturbations are, unfortunately, not suitably elaborated by a current process in such a way as to start 
parasitic crisis processes, for example, by superimposing increasing variations on running processes adopting over time significant interfering effects. The concept also includes any kinds of aggregated causes. Here we simply refer to them as causes.

Causes adopted giving rise to crises may be of different types.

For instance causes may come in any combinations:

- Accidental, unintentional and having no other effective explanations for their occurrence, except chance. This cause may have a brief or undefined temporal duration.

- Persistent, the cause persists over a long time-span, in proportion to the duration of the hosting process. Its persistence is required to maintain the crisis process. Another situation occurs when non-persistent causes produce configuration changes having long term effects.

- Temporary, the cause persists for a short time-span, proportional to the duration of the hosting process. Its persistence is not required to maintain the initiated process of crisis. Another situation occurs when the cause produces configuration changes having temporary effects.

- Emergent, self-acquired, unexpectedly the interaction and the interfering of on-going processes, possibly perturbed, make emergent a new process, in this case a process of crisis. A case may occur when on-going processes generate perturbing side effects. Side effects can establish an additional cause or be additional inputs to be elaborated by the on-going processes.

The concept of cause may be considered in different ways within linear contexts (LCs) or non-linear contexts (NLCs).

In LCs a cause may be considered as any parametrical variation within the process and as having linear outcomes.

In NLCs and their combinations with LCs a cause may be considered as any parametrical variation within the process and as having non-linear outcomes.

Malfunctioning in emergent processes may be caused by the occurrence of defects or lack of stability of the initial conditions, e.g., resources available, environmental situations, or external perturbations.

The acquisition of the nature of emergence by a process of on-going sequences of steps toward a crisis may be caused by the acquisition of synchronisation(s) and coherence in correlation with those of external perturbations, environmental situations, or the cyclic nature of side-effects.

Actions on causes, such as their removal or changing, may have expected outcomes except when the crisis process activated becomes autonomous and independent from the initial cause.

When the process of crisis is due to the continuous presence of a cause, then some regulatory hypotheses are plausible.

In the following, we briefly mention some possible reorienting (as opposed to regulatory) approaches when the crisis processes are assumed to have possibly acquired properties such as having adaptive, reconfiguring, restoring, and self-repairing abilities due, for instance, to robustness of coherence having several different means of self-sustenance.

\section{Actions on dominance}

The experimenter should have tools available to detect and reveal the dominant components of the process of crisis. In conceptual accordance with the search for order parameters in Synergetics (Haken 1988), we consider here the possibility of finding the possible dominant ones in specific crises. More generally, it may be of help, for instance, to consider possible statistical properties (Georgy et al., 1016) detected using suitable techniques such as Principal Components Analysis (PCA) (Jolliffe, 2002; Vidal and Sastry, 2016), Recurrence Quantification Analysis (RQA), Multivariate Data Analysis (MDA), Cluster Analysis mentioned above, , Time-Series Analysis (Box et al., 2015), Pearson Product Moment Correlation Coefficient (PPMCC) (Rupp and Walk, 2007) . 
However, besides the hypothesis of detecting the dominant process imposing, for instance, linearity or non-linearity, complexity properties such as power laws, network properties, scale invariance, metastructural properties, or evolution in general, one may consider how and why the dominating process is established.

Since such multiple processes are assumed to interact and interfere amongst themselves, the dominant one may even be considered as being given by the processes of interaction and interference themselves. While actions on the dominant one, intended as the consequent, resulting, in some way acquired one, are expected to have consequent cascade effects, in turn, actions on interactions and interference are expected to have effects on its dominance, possibly affecting the occurrence and identification of the dominant process itself.

\section{Actions on steps}

Local actions on steps of the crisis process usually have local reparative purposes.

Supposed regulatory, modifying, and restoring actions on steps are usually the preferred options, being the more intuitive and urgent to deal with current negative effects.

Local modifying interventions may be intended as adjusting local values as if they came from occasional and unfortunate deviations from the on-going regular processes in the hope of restarting the usual process, activating a return to normality.

However, this may positively perturb a process of emergent crisis or of malfunctioning emergence, even without guaranteeing suitable consequent, and desired regulatory effects. The strategy to reassess the process by acting on local steps may be based on various assumptions about the process, such as its linearity, its possible adaptive or self-restorative capabilities, and its effects on other interacting and interfering processes. In this last case, the desired effect could be to make other processes dominant.

\section{Actions through antagonistic interventions}

The strategy in this case is to contrast the process of crisis. This intervention may have the purpose of weakening, or extinguishing the crisis process with the hypothesis that the crisis is the problem, with the purpose of clearing the field to allow (re-)emergence of other more welcome processes. The source of the unwanted crisis process may remain unidentified and neglected once made inoffensive.

Otherwise the strategy is to change the game in some way, even though the new game cannot be completely decided.

\section{Strengthening actions on the hosting process}

In this case there is the working assumption that the clearly distinguishable crisis process can parasitize the hosting one because of the latter's weakness. In the case of non-linear relationships between the two processes, the strengthening action may make the original process more robust and able to resume its evolutionary path. If the process of crisis can be considered as a deviation, as a degeneration of the original one, this approach is counterproductive since such strengthening will affect both processes.

\section{Actions through invasive corrective interventions}

The strategy in this case is to invasively change the crisis process. The assumption is that the process can be distinguished from the original hosting processes.

This simpler, invasive intervention consists of artificial changes to steps of the crisis processes as introduced above.

Possible interventions may be assumed to have perturbing finalised purposes such as the start of other contextual contemporary, interfering processes having the desired nature, trying to make them dominant.

As mentioned above, other kinds of intervention may be environmental by changing, for instance, the energy available, or by inserting suitable local, noise, perturbations, and impurities from the usual 
environment. Other possible interventions may consist of invasive recovering interventions finalised for imposing new complete subsequent initial conditions rather than acting on single steps.

\section{Actions through incompatibilities}

This approach is conceptually based on possible well-known incompatibilities such as acting upon deflation, inflation, increase in interest rates, and increase in employment in economic systems.

We consider here incompatibilities as possible constraints for the appearance or reduction in the degrees of freedom of crisis processes.

It is possible to insert incompatibilities, for instance, by suitably changing sequences of steps of the crisis processes $a$ s if the crisis process were different, e.g., liquidity injections in economic crises, from the desired one (in this case actions on steps are not reparative but finalised to give a different orientation to the crisis process itself); by acting on the original processes to make them incompatible from a certain point onwards with the established parasitic, possibly emergent, process of crisis, e.g., by inserting faster or slower modifications or temporary convergences.

This also has the purpose of reducing possible correlations among processes of crisis making them less robust.

A strategy may be finalised to change the nature of the processes of crisis, for instance, by inserting aspects of linearity in NLCs and deactivating properties or emergence of processes of crisis such as coherence, correlation, validity of scale invariance and power laws.

\section{CONCLUSIONS}

We have tried to introduce a more precise understanding of what a process of crisis is and how it can benefit from more suitable possible modifying interventions. Taking into account a suitable level of representation, considering various possibilities, such as the crisis as a parasitic non-autonomous process, as an autonomous process, as a convergent process, as being given by coherent, subsequent and related new degenerative properties or loss of coherence among emergent processes of the hosting one(s). We have outlined possible symptoms for the diagnosis and prediction of processes of crisis, presenting various examples and types of crises.

In the section ACTIONS ON CRISES we proposed some generic types of actions on crises, possibly combined, such as actions on causes, on dominant processes, on steps in the crisis, strengthening actions on the hosting process, adopting antagonistic interventions against the crisis, adopting invasive corrective interventions, as well as the usage of incompatibilities.

The main purpose of this article was to outline how there are different types of processes of crisis having different natures and how suitable approaches should be adopted, by avoiding standardised generic interventions assumed to have general effectiveness.

Systems and processes of crisis may be of different types even though their complexity may be shared. Further research should consider suitable modelling allowing simulations.

\section{RÉFÉRENCES}

Biswas, A., Mandal, S.J., \& Kumar, V.R. (2014). Processing, Properties \& Applications of Ferroelectric Material: Ferroelectricity,Material Processing, Curie Temperature, Single Crystal. LAP Lambert Academic Publishing, Saarbrücken, Germany.

Box, G.E.P., Jenkins, G.M., Reinsel, G.C., \& Ljung, G.M. (2015). Time Series Analysis: Forecasting and Control. Wiley, Hoboken, NJ.

Bunge, M. (2001). Philosophy in Crisis: The Need for Reconstruction. Prometheus Books, Amherst, NY.

Buzsáki, G. \& Christen, Y. (Eds.). (2016). Micro-, Meso- and Macro-Dynamics of the Brain. Springer, New York, NY, USA. 
Christensen, K., \& Moloney, N.R. (2005). Complexity and Criticality; Imperial College Press, London, UK.

Dash, M. (2010). Tulipomania: The Story of the World's Most Coveted Flower and the Extraordinary Passions it aroused. Broadway Books, New York, NY, USA.

Diamond, L., \& Plattner, M.F. (Eds.). (2015). Democracy in Decline? Johns Hopkins University Press, Baltimore, MA.

Fannes, M. Maes, C., \& Verbeure, A. (Eds.). (1994). On Three Levels: Micro-, Meso-, and MacroApproaches in Physics. Springer-Verlag, New York, NY, USA.

Franzini, E. (2015). Filosofia della Crisi. Guerini e Associati, Milan, Italy.

Friedenberg, J.D., \& Silverman, G.W. (2015). Cognitive Science: An Introduction to the Study of Mind. SAGE Publications Inc., Los Angeles, CA, USA.

Georgy L., Shevlyakov, G., \&, Oja, H. (2016). Robust Correlation: Theory and Applications. Wiley, Chichester, UK.

Gorbana, A.N., Smirnovab, E.V., \& Tyukinaa, T.A. (2010). "Correlations, risk and crisis: From physiology to finance". Physica A, 389: 3193-3217.

Haken, H. (1988). Information and Self-Organization. A macroscopic approach to complex systems. Springer, Berlin.

Haunss, S. (2015). Conflicts in the Knowledge Society. Cambridge University Press, Cambridge, UK.

Haykin, S.O. (2008). Neural Networks and Learning Machines. Pearson, Cambridge, UK.

Jolliffe, I.T. (2002). Principal Component Analysis. Springer-Verlag, New York, NY, USA.

Kumar, K.(2004). From Post-Industrial to Post-Modern Society: New Theories of the Contemporary World. Blackwell Publishers, Oxford, UK.

Licata, I. (2008). Emergence and Computation at the Edge of Classical and Quantum Systems. In Physics of Emergence and Organization; (Licata, I., Sakaji A., Eds.), World Scientific, Singapore: 125 .

Licata, I. (2010). Living with Radical Uncertainty. The Exemplary Case of Folding Protein. In Crossing in Complexity: Interdisciplinary Application of Physics in Biological and Social Systems; (Licata, I., Sakaji, A., Eds.), NovaScience, New York, NY, USA: 1-9.

Licata, I. \& Minati, G. 2016). "Emergence, Computation and the Freedom Degree Loss Information Principle in Complex Systems". Foundations of Science, 21 1-19.

Liljenstrom, H. \& Svedin, U. (2005). Micro Meso Macro: Addressing Complex Systems Couplings. World Scientific Publishing. Singapore.

Minati, G. \& Pessa, E. (2018). From Collective Beings to Quasi-Systems. Springer, New York, NY, USA.

Minati, G. (2012a). "Knowledge to manage the Knowledge Society". Learn. Organ., 19: 352-370.

Minati, G. (2016a). "Knowledge to Manage the Knowledge Society: The Concept of Theoretical Incompleteness". Systems, 4: 1-19. http://www.mdpi.com/2079-8954/4/3/26/pdf

Minati, G.,(2016b). "Democracy as consensus? The case of artificial consensus". Acta Europeana Systemica, 6, http://aes.ues-eus.eu/aes2016/12 Minati-Gianfranco EN.pdf

Minati, G. (2006). Some Comments on Democracy and Manipulating Consent in Western PostDemocratic Societies. In Systemics of Emergence: Research and Applications, (Minati G., Pessa E., Abram M., Eds.), Springer, New York, NY, USA: 569-584.

Minati, G. (2015).The Social field designed by Architecture. In Systemic continuities and interactions between architecture and social systems: Knowledge for the future of the knowledge society, Proceedings of the $2^{\text {nd }}$ European Systemics Seminars of the European Union for Systemics (EUS), Brussels, Belgium, 16 October; (Claeys, D. Ed.), Acta Europeana Systemica, 5, http://aes.ueseus.eu/aes2015/08_Minati-Gianfranco_EN.pdf 
Minati, G., (Ed.). (2012b). Special Issue: Knowledge to manage the Knowledge Society. Learn. Organ., 19(4): 296-382.

Minati, G. \& Pessa, E. (2006).Collective Beings; Springer: New York, NY, USA.

Nescolarde-Selva, J.A. Usó-Doménech, J.-L. \& Gash, H. (2014). "A theorical point of view of reality, perception, and language". Complexity, 20: 27-37.

Paxton, M., Kolpinskaya, E., \& Jonasova, J. (Eds.). (2015). Politics in Crisis? Cambridge Scholars Publishing, Cambridge, UK,.

Pessa E., (2008). Towards a general theory of change. In Processes of emergence of systems and systemic properties. Towards a general theory of emergence; (Minati, G., Pessa, E., Abram, M., Eds.),World Scientific, Singapore: 603-24.

Pessa, E. \& Penna, M. P. (1994). Catastrophic Interference in Learning Processes by Neural Networks. In ICANN '94: Proceedings of The International Conference On Artificial Neural Networks; (Marinaro, M., Morasso, P.G., Eds.), Springer, New York, NY, USA:589-592.

Rasband, S.N. (2015). Chaotic Dynamics of Nonlinear Systems. Wiley, New York, NY, USA.

Rupp, A.A., \& Walk, M.J. (2007). Pearson Product-Moment Correlation Coefficient, In Encyclopedia of Measurement and Statistics; (Salkind, N. J., Ed.), Sage, London, UK: 1023-1027.

Saunders, P. (2010). An Introduction to Catastrophe Theory. Cambridge University Press, Cambridge, New York, NY, USA,

Shaikh, A. (2016). Capitalism: Competition, Conflict, Crises. Oxford University Press, Oxford, UK,.

Solé, R.V. (2011). Phase Transitions. Princeton University Press, Princeton, New Jersey.

Vidal, R., Ma, Y., \& Sastry, S.S. (2016). Generalized Principal Component Analysis. Springer, New York, NY, USA.

Walby, S. (2015). Crisis. Polity Press, Cambridge, UK.

Yang, S.-Q. (2016). Strength Failure and Crack Evolution Behavior of Rock Materials Containing Pre-existing Fissures. Springer, New York, NY, USA. 